\title{
Eficacia de cápsulas radiales en la promoción de alimentación saludable
}

\author{
Efficacy of radio capsules in promoting \\ healthy eating
}

\begin{abstract}
The high prevalence of obesity in children supports the need to implement preventive actions such as the development of programs to promote healthy food intake. Objective: To evaluate the effectiveness of radio capsules in developing a positive attitude towards healthy eating in children. Method: Under an experimental design with control group, were assessed using a scale attitudinal differences bifactorial pre and post intervention in two groups. The average age of each group was 10 years. In the experimental group 28 children participated in the preintervention assessment and 22 in the post intervention; in the control group 36 students participated in the pre-intervention assessment and 32 in post evaluation. Results:

The factor attitude toward healthy eating, and healthy behaviors decreased in the control group, while in the experimental group there was a slight increase in the average factor both in attitude and behavior factor. The only statistically significant differences between the averages of the measurements before and after the intervention in the control group, were in both attitude factor ( $t$ (66) $=-3.108, p=.003$ ) compared to the behavior factor ( $t$ (65) $=-3.350, P=.001)$. Conclusions: Students exposed to radio capsules had increased awareness of healthy eating, while the control group decreased significantly positive attitude towards healthy eating in both factors studied.

Key words: radio; obesity; quality of life.
\end{abstract}

Heleny Méndiz R. (1)

Alfonso Urzúa M. (2)

Lynda Álvarez R. (1)

Daniel Arce M. (1)

Daniel Vallejos S. (1)

Anibal Zamorano P. (1)

(1) Escuela de Periodismo, Universidad Católica del Norte, Antofagasta, Chile. (2) Línea de Investigación en Salud, Bienestar y Calidad de Vida, Escuela de Psicología, Universidad Católica del Norte, Antofagasta, Chile.

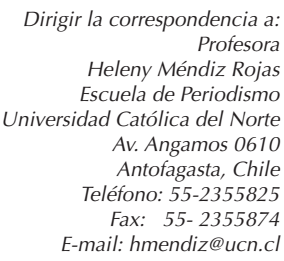

E-mail: hmendiz@ucn.cl

Este trabajo fue recibido el 28 de Mayo de 2013 y aceptado para ser publicado el 16 de Septiembre de 2013.

\section{INTRODUCCIÓN}

Desde el año 2002, la Organización Mundial de la Salud considera la obesidad como una epidemia mundial, con una prevalencia estimada de obesidad y sobrepeso en escolares de un $2,7 \%$ y $7,6 \%$ respectivamente (1). En Chile, el año 2000 se registraron 1.294.649 menores obesos $(2,3)$. La Junta Nacional de Auxilio Escolar y Becas, informó que el año 2009 21,8\% de los escolares presentaba obesidad y $22,4 \%$ sobrepeso, indicando un alza respecto al año 2006 (4). Diversos estudios nacionales han reportado prevalencias de sobrepeso que van de un 20 a un $22 \%$ y de un 3,7 a $23,8 \%$ de obesidad en escolares $(5,6)$. La prevalencia de obesidad es mayor en niños en edad prescolar, especialmente entre los 3 y 4 años de edad (7), siendo las tasas de prevalencia de sobrepeso y obesidad en escolares que ingresaban a $1^{\circ}$ básico, entre 1987 y el año 2000 semejantes a las de España y Estados Unidos (8). El año 2010, la obesidad afectaba al 9,9\% de los menores de 6 años, estando además un $22,4 \%$ de los niños con sobrepeso (9).

No existe una causa única para esta pandemia. En Chile, el modelo de desarrollo económico vigente ha cambiado el patrón de alimentación y actividad física. La ingesta aumentada de alimentos hipercalóricos, a la vez que la jornada escolar completa, la inactividad física y la opción por la comida rápida o alimentos altos en grasas, azúcares y/o sodio, de consumo y preparación rápida, así como su publicidad, estarían contribuyendo a la mantención del sobrepeso y la obesidad (10-13).

Diversos esfuerzos gubernamentales y no gubernamentales se han desarrollado en post de la prevención, tales como el Plan Nacional de Promoción de la Salud (4), la creación del Consejo Nacional de Promoción de la Salud, Vida Chile, a cargo del Ministerio de Salud (3), el desarrollo de la Estrategia Global contra la Obesidad- EGO, la Campaña 5 al día, dirigida a promover el consumo de frutas, verduras y hortalizas, entre otras. El último plan nacional fue lanzado el 2010, Ilamándolo campaña contra la obesidad "Elige vivir Sano", orientado a promover el consumo de alimentos saludables, la práctica deportiva al aire libre, además de la participación de la familia 
en la lucha contra la obesidad. Pese a estos esfuerzos, la meta bicentenario propuesta el año 2000 de reducir la obesidad entre escolares de seis años de un $16 \%$ a un $12 \%$, no logró cumplirse. Hoy, la meta sanitaria es reducir al 2020, la obesidad infantil, de un 20 a un 15\% (14).

La alta prevalencia y multicausalidad de este problema, ameritan la implementación de programas de promoción de la ingesta de alimentos saludables, especialmente en el segmento escolar, quienes, con la implementación de la Jornada Completa Escolar, pasan gran parte de su tiempo en los establecimientos educacionales. Es más efectivo cambiar hábitos de vida tempranamente para lograr un impacto duradero en donde cambios en los hábitos alimentarios, un aumento de la actividad física y una dieta sana y equilibrada se traducirán en un enfoque de éxito en el tratamiento de la obesidad (15).

Los hábitos alimentarios son producto de muchos componentes, siendo uno de ellos la actitud, es decir, la predisposición conductual con que enfrentan los niños la alimentación y la vida sana. Todo individuo, en su infancia, ha estado bajo el proceso de socialización primaria dado por sus padres, sin embargo, a partir del segundo ciclo básico, es más relevante la socialización secundaria. En esta etapa, los menores adquieren nuevas bases, externas al círculo íntimo, que luego se convertirán en actitudes y que son el resultado de la comunicación con sus pares, profesores y lo que lograr aprehender de los medios de comunicación. Es allí, donde la radio juega un rol como elemento incentivador de actitudes en el ambiente de socialización secundaria, en donde las posibilidades de apoyarnos en lo auditivo para reforzar los procesos de aprendizaje son más amplias de lo que nos marca un entorno mayoritariamente visual como es la televisión (16).

El objetivo de esta investigación, fue evaluar la eficacia de cápsulas radiales, en el cambio de actitud hacia la alimentación saludable en niños de enseñanza básica de colegios municipalizados de la ciudad de Antofagasta. Se parte de la hipótesis que el grupo expuesto a la emisión de cápsulas radiales con contenidos preventivos cambiará de actitud de manera positiva hacia la alimentación sana.

\section{SUJETOS Y METODO} Participantes

Se consideró para la intervención a menores escolarizados (alumnos de quinto año básico) de dos escuelas municipalizadas de la ciudad de Antofagasta, una de las cuales fue sometida a la exposición de las cápsulas radiales. Los establecimientos fueron elegidos aleatoriamente del universo de escuelas básicas municipalizadas de la ciudad. Considerando un 95\% de nivel de confianza, 95\% de poder estadístico, una precisión de 6, la varianza de la variable a estudiar en el grupo control de 23,40 y un porcentaje de pérdidas equivalente al $10 \%$ se requirió un mínimo de participantes de 19 sujetos por grupo. En el grupo experimental, participaron en la evaluación pre intervención 28 menores y en la evaluación pos intervención 22 . En tanto en el grupo control participaron en la evaluación pre intervención 36 estudiantes, y en la evaluación post 32 . La tabla 1 muestra el desglose de los participantes por sexo y la media de edad de cada grupo.

\section{Instrumentos}

Para medir la variable a predecir "actitud hacia la alimentación saludable", se utilizó una escala creada ad-hoc, en tanto se utilizó como variable predictora la emisión de cápsulas radiales con contenidos que promovían una alimentación sana.

La escala actitudinal se construyó en una etapa previa, con alumnos de enseñanza básica de otro establecimiento municipalizado, en donde a partir de una escala inicial de 46 preguntas, mediante el análisis de ítems y de la estructura factorial, se opta por una escala final de 12 ítems, estructurados en base a dos factores actitud frente a los alimentos y conductas saludables, los que explican un $44,3 \%$ de la varianza. El alfa de Cronbach para la escala total fue de .89 y de .83 y .80 para cada factor. La escala está construida con formato de respuesta tipo likert, con puntajes que van desde 1 a 5 dependiendo del grado de acuerdo o desacuerdo con cada reactivo, donde a mayor puntaje, mayor actitud favorable hacia una alimentación saludable y mayor presencia de conductas sanas. El puntaje se invierte en aquellos reactivos redactados en sentido contrario a la dimensión. La tabla 2 muestra los reactivos que componen cada factor.

La cápsula radial es un programa cuya principal característica es propiciar un cambio de actitud frente a algo. Se utilizan para la realización de campañas educativas y sociales. Su duración varía entre los 45" y los 3 minutos, tiempo durante el cual se entrega un mensaje determinado. Las cápsulas radiales fueron creadas por los participantes del curso "En la Onda de la Radio" del programa Desarrollando y Liderando Talentos Académicos de la Universidad Católica del Norte (DeLTA UCN). Para determinar si éstas cumplían con el propósito de fomentar una alimentación sana, fueron previamente evaluadas mediante un focus group en estudiantes de enseñanza básica, quienes formularon sus preferencias, junto con confirmar la comprensión de sus contenidos a nivel acústico y conceptual. De las 8 cápsulas, se eligieron 4 que cumplían con los requerimientos buscado por los investigadores.

\section{Procedimientos}

Una vez obtenido el consentimiento del Comité de Ética de la Universidad Católica del Norte, se establecieron los contactos con los distintos establecimientos que formarían

\section{TABLA 1}

Distribución de la muestra por sexo

\begin{tabular}{lcccc}
\hline Grupo & Hombres & Mujeres & Media edad & DE \\
Experimento pre & 17 & 11 & 10,85 & 1,20 \\
Control pre & 17 & 19 & 10,77 & 0,72 \\
Experimento post & 9 & 13 & 10,63 & 1,17 \\
Control post & 15 & 17 & 10,71 & 2,11 \\
\hline
\end{tabular}


parte de esta investigación, seleccionándose cuatro de estos: uno para la etapa de construcción de la escala, uno para la evaluación de las cápsulas radiales, uno como grupo experimental y el último como grupo control.

Cuatro cápsulas en total fueron grabadas en CD y expuestas al aire durante dos recreos diarios transmitidas en la radio del establecimiento educacional. Las ideas fuerza de los cuatro mensajes fueron: imaginarse un mundo sin comida chatarra; correr y disfrutar de una vida sana comiendo saludablemente; celebrando el día de la comida sin chatarra más la narración de un partido de fútbol entre los equipos Estrellas Chatarra vs. Comida Sana.

Las cápsulas fueron transmitidas durante 10 días hábiles consecutivos en el establecimiento elegido como grupo experimental, dos veces al día dentro de la jornada escolar, con una duración de 15 minutos para el primer recreo y de 10 minutos para el segundo. Para ello se hicieron 5 discos compactos (CD) equivalentes a cada día de la semana, en los cuales se intercalaron cápsulas radiales con música de escucha frecuente por los menores - previamente consultada a los encargados de la radio del establecimiento - teniendo una distribución diferente en cada ocasión. Se consideró que los menores mientras jugaban, comían su merienda o se juntaban con sus amigos, irían escuchando estas cápsulas. Los cuestionarios fueron pasados antes de comenzar con la emisión de las cápsulas radiales y una semana después de la última emisión.

\section{RESULTADOS}

El gráfico 1 representa las medias obtenidas por cada uno de los factores tanto en el grupo control con el experimental, en las evaluaciones pre y post intervención. Tal como se puede observar, tanto el factor actitud frente a la alimentación sana, como conductas saludables, disminuyeron en el grupo control (de 24,83 a 20,71 y 21,11 a 18,31, respectivamente), en tanto en el grupo experimental se observó un leve aumento en la media tanto en el factor actitud (de 18,79 a 20,50) y en el factor conducta (de 17,29 a 17,81). Sólo fueron estadísticamente significativas las diferencias entre las medias de las mediciones pre y post intervención en el grupo control, tanto para el factor actitud ( $t(66)=-3,108 ; p=.003$ ) como para el factor conducta $(t(65)=-3,350 ; p=.001)$.

\section{DISCUSIÓN}

La investigación realizada tuvo por efecto evaluar la eficacia de las cápsulas radiales en la promoción de una alimentación saludable. Se consideró como eficacia a la capacidad que tiene una intervención para producir cambios en una dirección esperada, que sean claramente superiores a la no intervención o a la de otras intervenciones disponibles (17). En este contexto, se observó un incremento positivo de las medias en los resultados del grupo experimental tanto en la actitud favorable hacia una alimentación sana, como en la presencia de conductas saludables. Al no ser estas diferencias estadísticamente significativas, no podemos confirmar nuestra hipótesis referente a la eficacia de las cápsulas en el cambio de actitud favorable hacia la alimentación saludable, sin embargo, constituye un hallazgo, toda vez que suponemos que si se exponen los mensajes auditivos en un período de tiempo más extenso, podrían variar significativamente, dado que en esta oportunidad la intervención se centró en una campaña corta, pero intensa.

Podemos suponer que a mayor tiempo de exposición a las cápsulas, podrían acrecentarse esta diferencia. Si comparamos ambos grupos, los estudiantes expuestos a las cápsulas radiales tuvieron mayor conciencia acerca de la alimentación saludable, en tanto los del grupo control aumentaron significativamente la actitud negativa frente a la alimentación sana en ambos factores estudiados.

Los resultados encontrados sugieren profundizar en aqueIlos elementos que pudiesen afectar la eficacia, siendo uno de estos el corto tiempo de exposición de las cápsulas, dado que resulta más complicado reforzar un cambio de actitud positivo que uno negativo. De acuerdo a esto, futuras investigaciones podrían ampliar el tiempo de transmisión de los mensajes radiales con el fin de obtener evidencia significativa que explique una tendencia positiva o negativa a largo plazo, reflejando la eficacia de las cápsulas.

Dado este corto tiempo de exposición, se debe sintetizar de manera casi perfecta su contenido, para poder conseguir que el mensaje que se busca transmitir haga sentido en las personas que lo escuchan y las invite a la reflexión. Debido a la influencia de los medios de comunicación en la sociedad - especialmente en edades tempranas - como modelo

TABLA 2

Reactivos de la escala de actitud hacia la alimentación por factor.

Factor

Actitud frente

a los alimentos
Reactivos

Me gusta más la bebida que el agua.

Me gustan los negocios de comida rápida como McDonalds, Doggis u otros.

Prefiero ir al patio de comidas del Mall que comer en casa

La leche es sólo para los bebés.

Cuando llego a casa, me instalo en el computador hasta que me llamen a comer.

Prefiero comer una papa frita que una fruta.

Comer chatarra está de moda.

Conductas

saludables
Tomo leche todas las mañanas.

Cuando llego a casa, me instalo frente a la televisión hasta que me llamen a comer. Me gustaría hacer deporte.

Es más entretenido jugar con mis amigos, que estar en casa jugando en el computador. Prefiero ver televisión que salir a jugar con mis amigos. 
de referencia en base a rostros y/o personajes populares, el reforzamiento de las ideas y mensajes va enfocado en la utilización de los mismos como elemento de reafirmación y ejemplo a seguir, ya que, pese a la cercanía de los niños para con otros niños en cuanto a las cápsulas radiales, los personajes públicos tienen mayor incidencia en el proceso de socialización secundaria.

En el caso de ser creadas por niños, deben ir enfocadas en estilos contemporáneos que tienen mayor aceptación, ello bajo el uso de cápsulas familiarizadas auditivamente con artistas del momento, creando un vínculo actitudinal de orden afectivo, ya que los niños serán capaces de sentirse identificados, promoviendo una disposición activa a oír el mensaje en su totalidad (18). Además, la repetición constante creará automáticamente, un efecto de memorización musical de las cápsulas, haciendo que sean identificadas e instaladas permanentemente en la memoria del público, especialmente, de los niños.

Creemos que este estudio preliminar aporta datos para seguir profundizando en la utilización de medios no tradicionales de educación (como lo es la radio escolar), dado el potencial educativo que pueden presentar las cápsulas, lo cual es especialmente conveniente al momento de generar cambios en las actitudes y favorecer así la salud de nuestra población infantil. Se debe considerar además que la utilización de mensajes radiofónicos contribuirá a desarrollar diversos aspectos en los niños. Desde lo pedagógico estudios afirman que la transmisión de contenidos didácticos mediante manipulación de sonidos refuerza la adquisición de conocimientos y aptitudes, al tratarse de un modo distinto de penetración de los mismos, siendo válidos tanto para contenidos curriculares como para actitudes y valores (16).

La fundamentación pedagógica que sustenta el uso de la radio en la educación son los mismos que justifican el empleo didáctico del resto de los medios de comunicación: la acusada presencia social de la comunicación audiovisual y la necesidad subsiguiente de favorecer una lectura crítica y creativa de los mismos, así como abrir la realidad educativa a la realidad del entorno. Algunas de las características que lo hacen recomendable para tareas educativas son que permite el trabajo colectivo, al ser un canal exclusivamente sonoro, favorece el valor de lo verbal, fomenta el diálogo, la discusión para trabajar la lectura crítica de sus mensajes y favorece también la adquisición de hábitos de investigación, iniciativa creadora, autogestión y autocuidado, entre otros (19).

Educar a través de la radio no suple a la escuela sino que la comunicación auditiva puede complementar lo aprendido tanto en el aula como en otros ambientes, por lo que el reforzar mensajes mediante la utilización de programas radiales de corta duración, transmitidos en las emisoras escolares, puede generar cambios en las audiencias.

\section{RESUMEN}

La alta prevalencia de obesidad en escolares de 10 años en promedio de edad, fundamenta la necesidad de implementar acciones preventivas como el desarrollo de programas de promoción de ingesta de alimentos saludables. Objetivo: Evaluar la eficacia de las cápsulas radiales en el desarrollo de una actitud favorable hacia la alimentación saludable en menores escolarizados, mensajes transmitidos en la radio del establecimiento educacional. Método: Bajo un diseño experimental con grupo control, se evaluaron mediante una escala actitudinal bi factorial las diferencias pre y post intervención en dos grupos similares, uno de ellos sometidos a cápsulas radiales. El promedio de edad de cada grupo fue de 10 años. En el grupo experimental, participaron en la evaluación pre intervención 28 menores y en la evaluación pos intervención 22; en tanto en el grupo control participaron en la evaluación pre intervención 36 estudiantes, y en la evaluación post 32. Resultados: tanto el factor actitud frente a la alimentación sana, como conductas saludables, disminuyeron en el grupo control, en tanto en el grupo experimental se observó un leve aumento en la media tanto en el factor actitud y en el factor

\section{GRÁFICO 1}

Medias por factor para los grupos control y experimental en mediciones pre y post intervención.
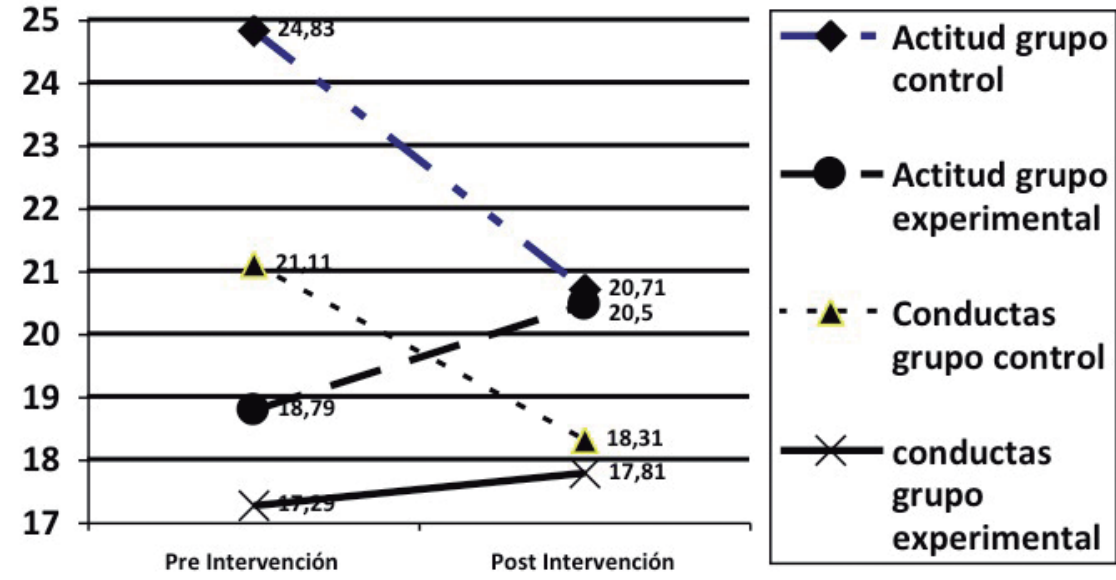
conducta; sólo son estadísticamente significativas las diferencias entre las medias de las mediciones pre y post intervención en el grupo control, tanto para el factor actitud $(t(66)=-3,108$; $\mathrm{p}=.003)$ como para el factor conducta $(\mathrm{t}(65)=-3,350 ; \mathrm{p}=.001)$. Conclusiones: Los estudiantes expuestos a las cápsulas radiales tuvieron mayor conciencia acerca de la alimentación saludable, en tanto los del grupo control disminuyeron significativamente la actitud positiva frente a la alimentación sana en ambos factores estudiados.

Palabras clave: radio; obesidad; calidad de vida.

Agradecimientos: Este estudio fue financiado por la línea de fondos concursables 2011 de la Vicerrectoría de Investigación de la Universidad Católica del Norte, la cual no tuvo injerencia alguna en el diseño del estudio, recolección, análisis o interpretación de los datos, preparación, revisión o aprobación del manuscrito.

\section{BIBLIOGRAFÍA}

1. Nutrition For Health And Development: A global agenda for combating malnutrition. Geneva. World Health Organization (Document WHO/NHD/00.6). Disponible en https://apps.who.int/nut/documents/nhd_mip_2000.pdf. [Consultado el 15 de agosto del 2013.

2. Atalah S, Eduardo Epidemiología de la obesidad en Chile. Rev Méd Clínica Las Condes 2012;23 (2): 117-23.

3. Vio del R F, Zacarías I, Lera L, Benavides MC, Gutiérrez AM. Prevención de la obesidad en escuelas básicas de Peñalolén: componente alimentación y nutrición. Rev Chil Nutr. 2011; 38(3): 268-76.

4. Crovetto M., Zamorano F, Medinelli S. Estado nutricional, conocimientos y conductas en escolares de kinder y primer año básico en 3 escuelas focalizadas por obesidad infantil en la Comuna de Valparaíso, Chile. Rev Chil Nutr. 2010; 37(3): 309-20.

5. Ulloa N, Sapunar J, Bustos P, Sáez K, Asenjo S, Taibo M, \& Cornejo A. Epidemiología del sobrepeso y obesidad InfantoJuvenil en las Comunas de Concepción, Coronel y Hualpén, VIII Región de Chile. Rev Med Chil. 2010; 138: 1365-72.

6. Loaiza S, Atalah E. Factores de riesgo de obesidad en escolares de primera año básico de Punta Arenas. Rev Chil Pediatr. 2006; 77(1); 20-6.

7. Kain J, Lera L, Rojas J \& Uauy R., Obesidad en preescolares de la Región Metropolitana de Chile. Rev Med Chil. 2007;
135: $63-70$

8. Eyzaguirre F, Mericq V, Ceresa S, Youlton R, Zacarías J. Prevalencia de sobrepeso y obesidad en niños que se controlan en pediatría ambulatoria en Clínica Las Condes. Rev Chil Pediatr. 2005; 76(2): 143-9.

9. Mañalich, J, Presentación indicadores de obesidad en la población chilena 2011. Disponible en página del Ministerio de Salud, http://www.minsal.cl/portal/url/ page/minsalcl/g_proteccion/g_alimentos/prot_ego.html. (consultada 13 de agosto 2013).

10. Busdiecker S, Castillo C \& Salas I. Cambios en los hábitos de alimentación durante la infancia: una visión antropológica. Rev Chil Pediatr. 2000; 71(1): 5-11.

11. Albala C, Vio F, Kain J, Uauy R. Nutrition transition in Chile: determinants and consecuenes. Public Health Nutr. 2002; 5(1a); 123-8.

12. Biblioteca del Congreso Nacional de Chile, Profundización del tema de la obesidad. ¿Por qué Chile se puso obeso?. 2007, disponible en http://www.bcn.cl/carpeta_temas/ temas_portada.2006-09-25.0806013222/documentos_pdf.2006-09-25.5168786990/area_1.2007-0213.2965927770 [Consultado el 13 de agosto 2013)

13. Reyes $M$, Díaz $E$, Lera L, Burrows R. Ingesta y metabolismo energético en una muestra de adolescentes chilenos con sobrepeso y obesidad. Rev Med Chil. 2011; 139: 425-31.

14. Gobierno de Chile. Página oficial del Gobierno de Chile: 2011. Gobierno Informa. Disponible en http://www.gob. cl/informa/2011/01/26/fijan-metas-de-reduccion-deobesidad-tabaquismo-y-consumo-de-alcohol-en-la-poblacion-escolar-para-el.htm [Consultado el 13 de agosto 2013)

15. Burrows R. Prevención y tratamiento de la obesidad desde la niñez: la estrategia para disminuir las enfermedades crónicas no transmisibles del adulto. Rev Med Chil. 2000; 128(1): 105-10.

16. Quintana-Garzón R. El lenguaje de la radio y sus posibilidades educativas. Comunicar 2001; 17: 97-101.

17. Ferro $R$, Vives $M$. Un análisis de los conceptos de efectividad, eficacia y eficiencia en psicología. Panace@ 2004, $V(16): 97-9$

18. Fleitas $A M$ \& Zamponi R. Actitud de los jóvenes ante los medios de comunicación, Comunicar 2002; 19: 162-9.

19. López-Noguero F. Reflexiones: los medios de comunicación en la educación social: el uso de la radio. Comunicar 2001: 141-8. 